\title{
FUKUSHIMA : UNE RECONSTRUCTION PÉRILLEUSE
}

\section{Cécile Asanuma-Brice}

Union rationaliste | «Raison présente »

$2021 / 4 \mathrm{~N}^{\circ} 220$ | pages 71 à 80

ISSN 0033-9075

DOI $10.3917 /$ rpre.220.0071

Article disponible en ligne à l'adresse :

https://www.cairn.info/revue-raison-presente-2021-4-page-71.htm

Distribution électronique Cairn.info pour Union rationaliste.

(C) Union rationaliste. Tous droits réservés pour tous pays.

La reproduction ou représentation de cet article, notamment par photocopie, n'est autorisée que dans les limites des conditions générales d'utilisation du site ou, le cas échéant, des conditions générales de la licence souscrite par votre établissement. Toute autre reproduction ou représentation, en tout ou partie, sous quelque forme et de quelque manière que ce soit, est interdite sauf accord préalable et écrit de l'éditeur, en dehors des cas prévus par la législation en vigueur en France. Il est précisé que son stockage dans une base de données est également interdit. 


\title{
FUKUSHIMA : UNE RECONSTRUCTION PÉRILLEUSE
}

\author{
Cécile Asanuma-Brice*
}

L'explosion de la centrale nucléaire de Fukushima Dai Chi en mars 2011 reste sans aucun doute l'une des catastrophes industrielles aux enjeux politiques cruciaux dans la période que nous traversons. Voici un monde lancé dans la recherche frénétique de sources énergétiques viables et respectueuses de l'environnement. Si l'industrie nucléaire fut présentée comme telle durant de longues années, les accidents successifs aux conséquences profondes et durables interrogent ce choix. La volonté politique, fondée sur un investissement industriel conséquent, de montrer que l'on serait capable de surmonter un tel accident a pourtant conduit le gouvernement japonais à décréter la réouverture totale de la zone évacuée autour de la centrale. Cette décision fut rendue possible par les recommandations de la CIPR (Commission internationale de protection radiologique) selon laquelle la limite d'exposition peut passer de 1 (seuil en temps normal) à $20 \mathrm{msv} / \mathrm{an}^{1}$ en période de reconstruction après un accident $^{2}$. Il s'agit là d'une première au monde qui n'est cependant pas sans conséquences humaines et matérielles. En premier lieu, cette reconstruction génère d'innombrables déchets dont il paraît important d'élaborer un bilan provisoire. Mais par-delà des débris, ce sont des questions éthiques fondamentales qui sont en jeu. Nonobstant celles que suscite la politique de communication sur le risque afin de stimuler une résilience qui n'irait pas assez bon train ${ }^{3}$, nous nous intéresserons ici à l'élaboration des méthodes planificatrices peu soucieuses de nos environnements de vie.

\footnotetext{
* Chercheuse CNRS, co-directrice de Mitate Lab Post Fukushima StudiesIRP CNRS

1 L'unité est le millisievert par an.

2 Ministry of the Environment Government of Japan (2013, updated on March 2017): Booklet to provide Basic Information Regarding Health Effects of Radiation.

3 Asanuma-Brice, C. (2015), «De la vulnérabilité à la résilience, Réflexions sur la protection en cas de désastre extrême. Le cas de la gestion des conséquences de l'explosion de la centrale nucléaire à Fukushima », Raisonpublique.fr : arts, politique, société, Raison-Publique.fr

Asanuma-Brice, C. (2021), Fukushima dix ans après. Sociologie d'un désastre, Maison des Sciences de l'Homme, 216 p. (p. 172-185).

Ribault, T. (2021), Contre la résilience. A Fukushima et ailleurs, Éditions L'Échappée.
} 
Ce travail de recherche est principalement fondé sur les documents de l'agence de reconstruction japonaise, ainsi que sur de nombreux terrains dans la zone depuis dix années ${ }^{4}$ qui nous ont permis d'effectuer des interviews non-directives auprès des habitants.

\section{LA POLITIQUE DE DÉCONTAMINATION ET LE TRAITEMENT DES DÉCHETS}

Au moment où s'ouvre le procès en appel des trois administrateurs de $\mathrm{TEPCO}^{5}$ en 2011, jusqu'à présent jugés innocents quant à la responsabilité de l'accident, TEPCO et le gouvernement affichent avec insistance leur responsabilité de reconstruction. Cette responsabilité-là s'accorde avec le discours très tôt tenu par le gouvernement japonais en faveur d'une réouverture de la zone ${ }^{6}$. Une décision qui s'est traduite par des campagnes drastiques de décontamination, soit le raclage des terres sur $5 \mathrm{~cm}$ et l'élagage des arbres jusqu'à $20 \mathrm{~m}$ en profondeur dans les forêts qui entourent les habitations comprises dans des «zones spéciales ". Ces périmètres avaient pour objectif de réduire l'exposition de la population aux débits de dose fixés par un programme spécifique à partir de janvier 2012. Le but n'était pas d'y atteindre les niveaux de rayonnement nucléaire d'avant l'accident, mais plutôt d'adapter la décontamination aux usages nécessaires du sol (Yasutaka \& Naito 2016) . Les méthodes utilisées $^{8}$ ont généré des centaines de tonnes de déchets ramassés dans des sacspoubelles et stockés dans un premier temps sur plus de 149330 sites répartis inégalement dans le paysage 9 . Les évaluations financières de ces mesures s'échelonnent entre 16 et 41 milliards d'euros (Evrard et al. 2019) ${ }^{10}$. À partir de 2016, le gouvernement définit trois sortes

\footnotetext{
4 L'autrice est résidente permanente au Japon.

5 Tokyo Electric Power Company, en charge de la gestion de la centrale au moment de l'accident et toujours en charge de son démantèlement.

6 L'annonce en a été faite en 2013 au moment où la décision d'attribuer l'accueil des JO au Japon a été proclamée suite à la déclaration du Premier Ministre Abe « Fukushima Nuclear Plant is under Control».

7 Yasutaka, T., Naito, W. (2016), « Assessing cost and effectiveness of radiation decontamination in Fukushima Prefecture, Japan ", Journal of Environmental Radioactivity, 151(2), Elsevier, January.

8 Op. cit. Asanuma-Brice, C. (2021), Fukushima dix ans après. Sociologie d'un désastre, Éd. MSH, p. 151.

9 Situation en déc. 2016. Cf. le document de l'agence de la reconstruction p. 4, https://www.reconstruction.go.jp/topics/main-cat1/sub-cat1-4/soukyoku/material/20191030-5_sokyoku-kaigo_kankyousho.pdf.

${ }^{10}$ Evrard, O., Laceby, J.-P., Nakao, A. (2019), " Effectiveness of landscape decontamination following the Fukushima nuclear accident: a review ", Soil, European Geosciences Union. SOIL, 5: 333-350.
} 
de déchets : ceux dont la teneur est inférieure à $8000 \mathrm{~Bq} / \mathrm{kg}^{11}$ qui seront réutilisés dans les travaux des ponts et chaussées, ceux dont la valeur radioactive est comprise entre $8000 \mathrm{~Bq} / \mathrm{kg}$ et $100000 \mathrm{~Bq} / \mathrm{kg}$ qui seront traités avec des méthodes spécifiques au lieu de stockage avant d'être réutilisés, et ceux qui dépassent les $100000 \mathrm{~Bq} / \mathrm{kg}$, qui seront rassemblés et stockés pour trente ans dans trois entrepôts : deux à Ôkuma et un à Futaba, communes adjacentes à la centrale. L'ensemble de ces déchets est estimé à 349000 tonnes fin juin 2021. Les controverses se concentrent actuellement moins sur l'efficacité de la décontamination (Evrard 2019) ${ }^{12}$ (limitée à des zones restreintes, hors zones forestières, impossibles à décontaminer bien que représentant $70 \%$ du territoire concerné) que sur le recyclage des déchets dont certains sont voués à être brûlés dans des incinérateurs, ce qui représenterait un danger supplémentaire pour le voisinage. Ce n'est pourtant qu'au prix d'une telle décontamination et du relâchement dans l'océan des tonnes d'eaux contaminées stockées autour de la centrale ${ }^{13}$ qu'il était envisageable de proposer la réouverture de la zone.

\section{ROUVRIR À TOUT PRIX : ÉTAPES POUR LA CRÉATION D'UNE AUTRE NORMALITÉ}

Cette décision, officiellement annoncée en juin 2017, s'est traduite par la levée de l'ordre d'évacuation sur les deux tiers de la surface non habitable, passant ainsi de $1100 \mathrm{~km}^{2}$ en avril 2011 à $300 \mathrm{~km}^{2}$ en 2021. Le gouvernement a annoncé son intention de continuer à étendre la levée de l'ordre d'évacuation selon un texte émis par le comité de reconstruction le 31 août 2021. Ce document stipule : « La nouvelle décision politique consiste à permettre aux résidents de vivre dans des zones où il est difficile de retourner chez eux, et à créer de nouvelles zones de récupération et de revitalisation en fonction du stade d'avancée de la réhabilitation des lieux ». Seuls six villages sont désormais concernés par ces mesures qui s'accompagnent d'une suppression des indemnités accordées aux réfugiés. Ce point est fondamental car la levée de l'indemnisation a contraint

\footnotetext{
${ }^{11}$ L'unité pour mesurer la matière est le becquerel par kilogramme.

${ }^{12}$ Evrard O. et al. (2019), art. cit.

13 1,37 millions de tonnes d'eau stockées actuellement dans 1000 réservoirs, comprenant encore du tritium (que l'on ne sait pas filtrer) et des traces d'autres nucléides (strontium 90, césium 134/137, etc.). En octobre 2021, un nouveau scandale a eu lieu car 27 des 30 filtres du système ALPS, censés filtrer cette eau, étaient troués. Voir le document de TEPCO avec les photos des filtres sur le site du METI, https://www.meti.go.jp/earthquake/nuclear/ decommissioning/committee/osensuitaisakuteam/2021/09/94-3-1-2.pdf.
} 
au retour une partie de la population qui n'a pas été en mesure de retrouver un emploi sur le lieu du refuge. Cela concerne une proportion importante des plus de soixante ans, nombreux dans la région, qui ont dû rentrer dans des communes désormais désertées par les populations les plus jeunes. En outre, les résultats de l'enquête de l'Agence de Reconstruction, rendus publiques en novembre 2020, montrent non seulement que, si l'on retire des calculs pour l'évaluation du retour les villages de Tamura et Kawauchi, dont seule une infime partie était sous ordre d'évacuation, le taux de retour tombe à $25 \%$ de rentrants, mais aussi que la nature du non-retour est moins liée à la peur de la radioactivité qu'à l'état des lieux après dix années d'abandon ${ }^{14}$. Les "rentrants" sont principalement des personnes âgées venues terminer leurs jours dans leur maison familiale. Force est de constater que l'accident nucléaire a été le catalyseur d'un processus déjà présent : le départ des populations les plus jeunes vers des zones plus urbanisées qui proposent de nombreux services et facilitent un quotidien parfois difficile dans les villages de montagne en cours de désertification ${ }^{15}$. La population rentrante reste donc extrêmement limitée. Comme remède à ce dépeuplement, les autorités ont mis en place un système de financements spécifiques pour toute personne se portant volontaire à venir s'installer dans la zone avec sa famille ${ }^{16}$. D'autres subventions sont attribuées à tout projet d'innovation qui y serait développé. Ces encouragements à redynamiser les lieux sont d'autant plus importants si les nouveaux arrivants proviennent de la région de la capitale, transformant la catastrophe en aubaine pour désengorger la métropole tokyoïte.

L'agence gouvernementale en charge a déterminé six zones de reconstruction prioritaires (ZRP) dans les six communes toujours évacuées, suivant imperturbablement un programme de reconstruction fixé en 2017. Comme symbole fort de remédiation, un espace circonscrit autour des gares (premières gares entièrement automatisées au Japon) a été rouvert pour les jeux olympiques en mars 2020.

La réouverture de ces ZRP est déterminée par un calendrier qui fixe les étapes par ordre : la décontamination, la destruction des bâtiments en ruine, la réouverture des principaux axes de circulation (routiers puis ferroviaires), la remise en activité des voies d'eau courantes et usées, la desserte électrique, etc. La reconstruction de lotis-

\footnotetext{
${ }^{14}$ Agence de la reconstruction, https://www.reconstruction.go.jp/topics/ main-cat1/sub-cat1-4/ikoucyousa/.

${ }^{15}$ Pataud-Célérier, P., Asanuma-Brice, C. (2021), « Fukushima, un douloureux retour ", Le Monde Diplomatique, mars.

${ }^{16}$ Op. cit. Asanuma-Brice, C. (2021), Fukushima dix ans après. Sociologie d'un désastre.
} 
sements habitables prend place dans les territoires préalablement partiellement décontaminés, dans lesquels les anciennes habitations ont été détruites. L'implantation de commerces, puis de services y est programmée.

Des lotissements ont été construits, sous forme de pavillons de banlieue bon marché, sur des sols totalement artificialisés. Malgré la volonté annoncée, lors des relogements dans les cités provisoires par village d'origine, de préserver les liens communautaires des habitants, l'obtention d'un nouveau logement dans les zones reconstruites fut finalement tirée au sort. La conséquence de cette méthode s'est traduite par le regroupement de foyers en provenance de l'ensemble des communes anciennement évacuées sans respect des liens de voisinage préexistants. Depuis 2019, des personnes cohabitent dans une de ces cités du village d'Ôkuma (commune qui accueille la centrale) pour un loyer de $194 € /$ mois. Ils sont logés dans des habitations de carton-pâte, en l'attente d'un relogement dans les logements collectifs en cours de construction plus près de la côte. D'autres pavillons sont construits pour les personnes désirant s'implanter plus durablement. Outre le fait que les habitants ne se connaissent pas, ils sont souvent dans un rapport de confrontation directe motivé par les inégalités de remboursement de leurs biens détruits par le désastre. Une ambiance de cité fantôme envahit donc ces lieux où personne ne se salue.

Ces zones planifiées côtoient d'anciens quartiers pavillonnaires, qui eux sont restés en l'état depuis l'accident (pavillons éventrés, herbes folles, et hot-spot à plus de $5 \mu \mathrm{sv} / \mathrm{h}^{17}$ ). Par ailleurs, la décontamination a parfois détruit totalement les paysages. Ici, l'intention concrète et positive de l'ingénieur est à opposer à l'in-attention simultanée portée à l'émotionnel. Des pans entiers de montagnes ont parfois été coupés, les arbres arrachés, la terre aplanie. Aucune prise en compte n'a été faite du rapport historique (au sens des histoires de vie) de l'être dans son milieu d'existence. Les habitants qui reviennent sont confrontés à un paysage qu'ils ne reconnaissent plus et cet aspect vient se rajouter au syndrome post-traumatique dont la majorité d'entre eux souffre encore (Tsujiuchi 2021) ${ }^{18}$. L'importance du rapport au territoire, aux lieux d'existence, est battue en brèche, et aucune considération n'a été apportée aux champs d'étude qui ont permis de l'appréhender. Pourtant la phénoménologie, à l'intérieur de laquelle se trouve la "mésologie " définie par Augustin

\footnotetext{
${ }^{17}$ L'unité est le microsievert par heure.

${ }^{18}$ Tsujiuchi, T. (2021), "Post-traumatic stress due to structural violence after the Fukushima Disaster », Japan Forum, 33(2): 161-188.
} 
Berque $^{19}$, propose des analyses et des concepts qui nous ont permis de comprendre l'importance du lien au paysage. Sa reconstruction via notre regard, lui-même composé par notre bagage de vie individuelle, attribue au milieu un sens relié à notre émotionnel ${ }^{20}$. Madame Eiko Kannô ${ }^{21}$, revenue vivre dans son village d'Iitate après plusieurs années passées dans les logements provisoires de Minami Sôma, exprimait ainsi son désarroi face à ses terres écorchées vives : "Toute cette terre contaminée raclée puis renfermée dans ces sacs, c'est une terre dont on a pris soin, que l'on a cultivée avec amour, toute notre vie. Elle est animée par les esprits comme le reste du vivant. Or, ils la déplacent comme si c'était une chose, un objet... » (Asanuma-Brice 202122).

La perception du planificateur, totalement extérieur et étranger au lieu qu'il planifie sans émoi et pour qui le territoire n'est qu'objet, diffère de celle de l'habitant pour lequel le territoire est le berceau de son quotidien, des années durant, créant ainsi un lien intérieur avec la terre qui l'accueille. Il s'agit là d'un enjeu important pour la planification urbaine. Elle échoue généralement, se laissant dominer par les rationalités économiques, permettant de fait la globalisation via l'urbanisation. Désormais, les zones d'habitats des parties planifiées des grandes villes du monde étonnent par leur ressemblance. Mues par les mêmes enjeux de rentabilisation, l'utilisation de matériaux peu chers, la création d'espaces " rationnels ", la construction ultra-rapide, répétable ad vitam, la bitumisation des espaces verts pour limiter l'entretien et, dans le cas présent, afin d'éviter la résurgence des nucléides et leur fixation par des végétaux (des terrains perméables sont plus facilement lessivables) deviennent prioritaires. Consécutivement, ces nouveaux espaces globalisés et globalisants créent une déconnexion du rapport à la terre, un désattachement, ou plutôt une dés-affection du lieu (au sens émotionnel de l'affectif, mais également en ce qu'on le dés-habite). Outre ce remodelage offshore de nos environnements, cette nouvelle façon d'habiter la terre transforme à nos yeux un milieu désormais externalisé et relégué au rang d'objet, qui plus est perçu comme un risque potentiel permanent.

\footnotetext{
${ }^{19}$ Berque, A. (2015), "La Mésologie pourquoi et pourquoi faire ? ", Annales de Géographie, 705: 567-579.

${ }^{20}$ Voir les nombreux travaux d'A. Berque sur le sujet et plus récemment Berque, A., Asanuma-Brice, A. (2021), " La destruction des paysages I \& II », Biocity... (en japonais).

${ }^{21}$ Interviewée en janvier 2020.

${ }^{22}$ Ibid. Asanuma-Brice, C. (2021), p. 186.
} 
Cette modification de l'occupation des sols, qui se traduit dans le cas que nous étudions par la réouverture complète de la zone, ne peut néanmoins s'affranchir des conséquences éventuelles liées à la réimplantation d'habitants dans des endroits encore inégalement contaminés. Pour ce faire, il est essentiel de considérer les aspects sanitaires de la catastrophe qui déterminent la pertinence du refuge et de la reconstruction.

\section{L'ÉPIDÉMIOLOGIE AU CENTRE DE L'ÉVIDENCE APODICTIQUE D'UNE APPROCHE MULTIDISCIPLINAIRE DE LA CATASTROPHE}

Les raisons pour lesquelles l'épidémiologie ne peut être absente de l'analyse sociologique et urbaine lors d'une nouvelle appropriation des territoires après un désastre nucléaire sont multiples. Au premier rang d'entre elles, la détermination de la question du refuge : c'est parce qu'un environnement sera désormais estimé épidémiologiquement impropre à la vie qu'il va être administrativement défini comme zone à évacuer. Évacuer un territoire est une décision aux conséquences graves. Il est donc important d'en mesurer la nécessité. Cette mesure du risque est en jeu dans les nombreuses controverses sur ce sujet complexe, une des grandes difficultés étant de déterminer les causalités souvent multifactorielles des cancers et autres maladies générés.

Il a fallu de multiples échanges avec les habitants de la région pour que la commission sanitaire, qui a déjà repéré 287 cancers de la thyroïde déclenchés parmi un échantillon de 370000 enfants de moins de 18 ans $^{23}$, ne cesse de continuer son travail d'observation qu'elle menaçait chaque année d'arrêter, arguant d'un sur-diagnostic dû au testage systématique ainsi que de l'amélioration des instruments de mesure médicale. L'argument de poids de certains épidémiologistes et des habitants, qui a finalement remporté cette confrontation, tient au fait que la plus forte croissance des maladies a été observée quinze années après l'accident à Tchernobyl ${ }^{24}$.

Outre les résultats publiés par la commission sanitaire, d'autres décès sont officiellement admis comme résultant des effets de l'accident nucléaire. Ils sont comptabilisés par la préfecture de Fukushima comme décès non consécutifs du tremblement de terre,

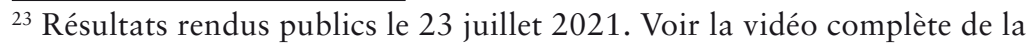
présentation de la commission sanitaire sur le site de OurPlanet (en japonais), https://www.ourplanet-tv.org/40600/.

${ }^{24}$ Shibata, Y. et al. (2007), « 15 years after Chernobyl: new evidence of thyroid cancer ", The Lancet, 358(9297): 1965-1966.
} 
mais relevant des " conséquences de la vie durant le refuge, des détériorations sanitaires et d'épuisement». Cela concernerait 2316 personnes ${ }^{25}$.

Il faut par ailleurs ajouter à ce bilan les 269 plaintes et demandes de reconnaissance pour maladies professionnelles déposées par les ouvriers de la centrale ${ }^{26}$. Plus récemment, en septembre 2021, deux travailleurs de la centrale sont décédés d'un cancer du pharynx dû à une surexposition reconnue par les autorités en charge ${ }^{27}$. Ces décès ont été rendus publics par voie de presse au Japon, plus rarement au-delà de ses frontières. Outre ces cas anonymes, et parce qu'en sciences sociales nous travaillons avec les habitants que nous suivons dans leur quotidien, il est des données qui ne peuvent être comprises par l'unique prisme du quantitatif.

\section{HASEGAWA KENICHI, UN FERMIER COMME LES AUTRES}

Les bottes crissent dans la neige pour s'arrêter au hasard d'une motte de terre. Une main, la peau tannée par des années de labeur, porte une boîte blanche qui grésille. Elle s'approche du sol alors que les sons qui en émanent s'intensifient. La voix éraillée par de trop nombreuses interventions tentant de rendre publique le marasme de la catastrophe qui lui aura pris tous ses biens, un compteur Geiger à coups/seconde à la main, Monsieur Hasegawa mesure ses champs : « 6,2 microsievert/heure. C'est autour de ça... On nous dit que ça va baisser avec la décontamination. Il faudra au moins 20 ou 30 ans pour arriver à une demi-vie ${ }^{28} \ldots$... Nous sommes en 2011, quelques mois après l'accident. Monsieur Hasegawa était un fermier parmi les 7000 habitants du village d'litate. Un village de montagne retiré, mais avec la spécificité d'être étiqueté éco-village pour la variété et la qualité de sa faune et de sa flore ${ }^{29}$.

Fortement touché par les retombées radioactives, le village n'a pourtant été évacué qu'un mois après les faits, suite à la réévaluation

\footnotetext{
${ }^{25}$ Site de la préfecture de Fukushima, https://www.pref.fukushima.lg.jp/ sec/01010d/tenji297.html.

${ }^{26}$ Reuters (2020), "Centrale nucléaire de Fukushima, 269 indemnisations pour accidents du travail » (6 octobre).

${ }^{27}$ NHK (2021), «Centrale de Fukushima Dai ichi Première reconnaissance de morts par cancer du pharynx comme accident du travail » (8 septembre).

${ }^{28} \mathrm{La}$ demi-vie est le temps nécessaire pour que la moitié des atomes se désintègre naturellement.

${ }^{29}$ Asanuma-Brice, C. (2018), «Fukushima, l'impossible retour dans les villages de l'ancienne zone d'évacuation : l'exemple d'Iitate ", GéoconfluencesENS Lyon, http://geoconfluences.ens-lyon.fr/actualites/eclairage/fukushuma-iitate-impossible-retour.
} 
de la zone évacuée en avril 2011. En mai 2011, Kenichi Hasegawa, alors âgé de 58 ans, prend la décision d'envoyer ses 12 vaches laitières à l'abattoir. Ses larmes coulent, sans un mot, les visages fermés se dissimulent au regard trop indiscret de la caméra de Doï Toshikuni venu fixer ce moment dans un film documentaire sur le village ${ }^{30}$. Kenichi Hasegawa participait avec ferveur à l'ensemble des réunions sur la gestion de l'accident. Des photos l'affichaient avec un rapport sanitaire entre les mains sur lequel il avait inscrit qu'il refuserait de servir de cobaye pour les médecins en charge d'établir la radioprotection de la zone. Présentant son parcours aux réunions annuelles des villageois, il avait pourtant décidé en 2017 de revenir vivre au village s'occuper de la maison familiale, tout en interdisant à ses enfants de le suivre. Parce qu'il fallait continuer à aller de l'avant, il avait même accepté de prendre part aux expérimentations agricoles visant à voir ce qui pouvait être cultivable sur des sols dont la contamination reste instable. Il avait entamé la culture du sarrasin, qui malgré ses efforts répétés, refusait de germer suite à l'enlèvement de la couche arable par les mesures de décontamination. "Rien à faire ça ne pousse pas! " maugréait-il avec un sourire venant souligner l'ironie du sort. Et l'ironie a pris forme. C'était en mars 2021. Alors que tous se mobilisent pour faire connaître leur situation contre le rouleau compresseur de la politique de reconstruction vaille que vaille, et contre la communication du risque qui l'accompagne intensifiée par l'ouverture des jeux olympiques, eux aussi maintenus en pleine pandémie, vaille que vaille, la nouvelle tombe : Kenichi Hasegawa a déclenché un cancer de la thyroïde. Fatigué par des centaines de présentations publiques et interviews diverses durant ces dix années qui ont suivi le désastre nucléaire, il refuse que son état de santé soit rendu public.

Rassuré par les rapports des experts affirmant avec confiance que "le cancer de la thyroïde n'est pas très mortel ", Hasegawa Kenichi se lance dans un nouveau combat pour la vie. Ce sera le dernier. Il s'achèvera par son décès le 23 octobre 2021, un peu plus de six mois après que son cancer a été détecté.

\footnotetext{
${ }^{30}$ Doi, T. (2012), Iitate, chronique d'un village contaminé, documentaire distribué par Echo-Echanges.
} 


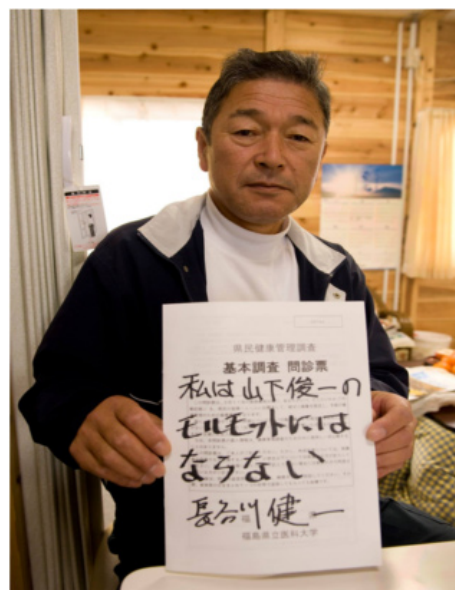

M. Hasegawa Kenichi en 2012, montrant le rapport de la commission sanitaire de l'université médicale de Fukushima chargée d'évaluer le nombre de cancers de la thyroïde sur lequel il avait écrit : "Moi, je ne serai pas le cobaye de Shunichi Yamashita (Docteur en charge de cette commission à l'époque). Signé : Hasegawa Kenichi ". Monsieur Hasegawa est décédé d'un cancer de la tyroïde le 23 octobre 2021. (Photo prise en 2012 dans son logement provisoire).

\section{À LA RECHERCHE D'UN PARADIGME PERDU}

Cette analyse qui part de la gestion administrative et urbaine du désastre pour arriver au cas particulier met en évidence une démarche dont le but n'est pas l'élimination des causes de la catastrophe, mais l'administration de ses conséquences. Ce «faire au mieux » vise à l'ouverture vers la possibilité de la continuation de l'activité à l'origine du désastre et de son acceptation. Il requiert une adaptation contrainte et construite par les diverses mesures en charge d'élaborer la résilience. La catastrophe de Fukushima n'est qu'un exemple parmi d'autres qui permet de mettre en exergue les contradictions de l'« évolutionnisme démocratique $»^{31}$ prétendant faire place aux variations les plus diverses d'êtres, de positions et d'opinions et qui, dans les faits, s'applique à éliminer divergences et conflits, pour s'orienter vers une solution unique. Ainsi, loin de permettre la reconsidération attendue du modèle de la société de consommation d'après-guerre, qui proposait pour axiome la famille nucléaire, l'urbanisation infinie et la destruction qu'elle induit pour les écosystèmes, Fukushima va corroborer ce qui tend à devenir un archétype de nos sociétés néolibérales ${ }^{32}$. Force est ainsi de constater que les catastrophes industrielles, en ce qu'elles alimentent l'ancien, ou parce qu'elles se limitent à des actes de victimisation passive, ne généreront pas les impulsions qui nous permettront l'élaboration d'un nouveau paradigme dont la nécessité semble pourtant désormais actée.

\footnotetext{
31 Stiegler, B. (2020), "Il faut s'adapter ». Sur un nouvel impératif politique, Collection NRF essais, Gallimard.

32 Kohs, S. (2021), Radiations et révolution. Capitalisme apocalyptique et luttes pour la vie au Japon, Éditions Divergences.
} 